\title{
A trajectory design method for RLV via artificial- memory-principle optimization
}

\author{
Hao $\mathrm{Li}^{*}$, and Changzhu Wei \\ School of Astronautics, Harbin Institute of Technology. Harbin 150001, China
}

\begin{abstract}
A trajectory optimization method for RLV based on artificial memory principles is proposed. Firstly the optimization problem is modelled in Euclidean space. Then in order to solve the complicated optimization problem of RLV in entry phase, Artificial-memory-principle optimization (AMPO) is introduced. AMPO is inspired by memory principles, in which a memory cell consists the whole information of an alternative solution. The information includes solution state and memory state. The former is an evolutional alternative solution, the latter indicates the state type of memory cell: temporary, short- and long-term. In the evolution of optimization, AMPO makes a various search (stimulus) to ensure adaptability, if the stimulus is good, memory state will turn temporary to short-term, even long-term, otherwise it not. Finally, simulation of different methods is carried out respectively. Results show that the method based on AMPO has better performance and high convergence speed when solving complicated optimization problems of RLV.
\end{abstract}

\section{Introduction}

Since the retirement of space shuttle, many countries start to develop the 2nd generation reusable launch vehicles (RLVs), which are more autonomous and reliable. After fulfilling its orbital tasks, RLV has to reentry the atmosphere. However, the reentry environment is very tough and complicated, a well-designed trajectory can bring about soft and tractable guidance commands, and make control easier to realize it. Accordingly, it is very important for RLV to seek a trajectory that satisfies all path constraints, such as heat rate, structural loads and dynamic pressure.

Generally, aerospace engineers use optimization algorithms to design different types of trajectories. Traditional optimization algorithms can find global optimum solutions in many cases [1], in which the objective functions and constraints are simple. Engineers expect the algorithms to be universal, nevertheless, it is not reality. When confronted with complicated optimizations, researchers realized that the emerging nature-inspired algorithms (NIAs), are more suitable with the help of high performance computers [2]. In order to achieve a much wider adaptability, these algorithms abandon mathematical or physical searching ways, and turn to imitate the behaviors of creatures or phenomenon in nature. Their searching process seems to be fuzzy or random, however researches showed NIAs have a global convergence.

\footnotetext{
* Corresponding author: lihao1800366@126.com
} 
These algorithms have a large family, including artificial neural network (ANN) [3-4], ant colony algorithm (ACA) [5], genetic algorithm (GA) [6], differential evolution (DE) [7] and particle swarm optimization (PSO) [8], and so on.

Biologists agreed that human is a kind of cleverest animal in nature, and studies point out that human's wisdom is closely related with memory. According to [9], if a NIA has the abilities of synergy, information exchange, diversity and evolution, and then it can solve a quite complicated problem. In the past decades, researches indicate that human memory principles can be transferred into a NIA [10-12]. They simplified the memory mechanism, and applied in different fields.

Based on the artificial memory principles, this paper proposed a NIA, which is called AMPO. There are three types of memory: temporary memory (TM), short-term memory (STM), long-term memory (LTM). A large number of memory cells build up the artificial memory system (AMS). In the process of optimization, a new alternative solution is firstly set to be TM, if the searching direction is "right", or the stimulus makes objective function lower, then the alternative solution turn to be STM. In the next search, if the same thing happens, then the alternative solution turn to be LTM, which has potential to be optimum solution, otherwise it turn back to be TM. As the search moves on, AMS will forget most alternative solutions or memory cells, and retain a small piece of the cells in LTM. The goal of trajectory design for RLV via AMPO is realized. Finally the convergence and validity of AMPO are verified through simulation.

This paper is organized as follows. Section 2 presents the optimization model of RLV. Section 3 develops the design of AMPO. Section 4 shows the simulation results. Section 5 contains conclusions.

\section{Problem Statement and Preliminaries}

\subsection{Problem statement of optimization}

In this paper, we consider the following optimization problem:

$$
\left\{\begin{array}{l}
\min f(\mathbf{X}) \\
\text { s.t. } \\
\quad g_{i}(\mathbf{X}) \geq 0 \quad i=1,2, \cdots, I \\
\quad \mathbf{X} \in S \in R^{n}
\end{array}\right.
$$

where $R^{n}$ is a n-dimensional Euclidean space; $\mathbf{X}=\left(x_{1}, x_{2}, \cdots, x_{n}\right)$ is a control vector; $S$ is a search space; $f(\mathbf{X})$ is the original objective function; $g_{i}(\mathbf{X})$ is the $i$ th inequality constraint. To make AMPO more adaptive, here the objective function can be rewritten as:

$$
F(\mathbf{X})= \begin{cases}f(\mathbf{X}), & g_{i}(\mathbf{X}) \geq 0 \\ F_{\text {max }}, & \text { others }\end{cases}
$$

where $F_{\max }$ is a man-made large number which is a punishment for an alternative solution that can not satisfy all constraints.

\subsection{Preliminaries of AMS}

According to theory of Ebbinghaus [13], the principles of memory is abstracted and viewed as a process of how brains (creatures) interact with the stimulus from the objective world. 
As it is shown in Fig.1, information from outside firstly enters into TS, when brain get a stimulus and it's good, information will be transferred into STM, otherwise the information will be forgotten gradually. It's the same with STM, good stimuli help brain to strengthen the memory of the information, then information turns to be LTM, and help creatures to build knowledge libraries and resolve similar or unknown problems (information). In addition, forgetting release the pressure of brains to memorize, it's helpful for creatures as well.

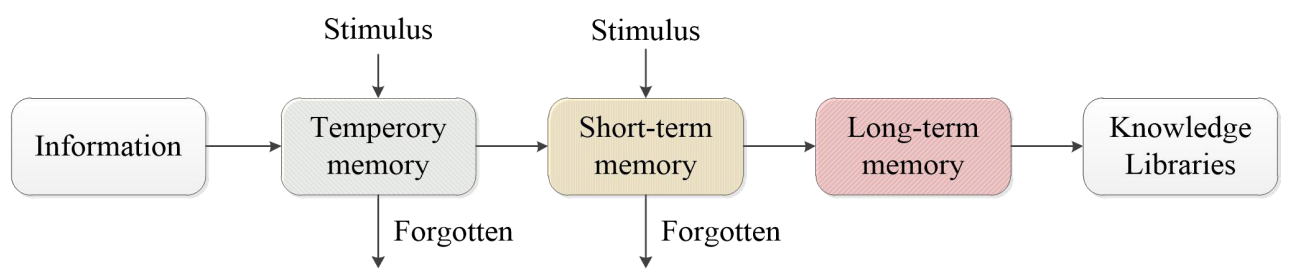

Fig. 1. The simplified model of memory.

\section{Construction of AMPO}

\subsection{Memory cell of AMS}

First of all, define the memory cell $M_{i}$ as below, which consists of an alternative solution $\mathbf{X}_{i}$, and its characteristics, namely

$$
M_{i}=\left(\mathbf{X}_{i}, m_{i}, t_{i}, s_{i}, f_{i}\right), i=1,2, \cdots, N
$$

where $m_{i}$ is the memory residual value; $t_{i}$ is the iteration running time; $s_{i}$ is the type of memory state, $s_{i}=T, S, L$ respectively corresponding to TM, SM and LM; if $f_{i}=0$, it means memory cell $M_{i}$ is not forgotten, if $f_{i}=1$, forgotten.

AMS includes $N$ alternative solutions $\left\{\mathbf{X}_{1}, \mathbf{X}_{2}, \cdots, \mathbf{X}_{N}\right\}$. In each iteration, it will practice all memory cells, in other words, it scans each alternative solution and calculates $F\left(\mathbf{X}_{i}^{t}\right)$, which represents the object function value that corresponds to the scanning (stimulus). There are two types of stimuli:

(1) If $F\left(\mathbf{X}_{i}^{t}\right)<F\left(\mathbf{X}_{i}^{t-1}\right)$, it means the stimulus are good for finding the optimum solution, and memory cell $M_{i}$ will strengthen memory, which makes $m_{i}$ bigger than before. The stimulus is so-called good stimulus.

(2) If $F\left(\mathbf{X}_{i}^{t}\right) \geq F\left(\mathbf{X}_{i}^{t-1}\right)$, it means the stimulus are useless, and $M_{i}$ will weaken memory, which makes $m_{i}$ smaller. The stimulus is so-called bad stimulus.

AMS scans all memory cells for a large number of iterations. Finally, this enables some memory cells with better characteristics of optimum to be strengthen-memorized, and some memory cells with worse characteristics to be forgotten.

\subsection{Evolution strategies of alternative solutions}

The position of $\mathbf{X}_{i}$ has some relationship with others while iteration going. Remark the position of all alternatives as $\mathbf{X}_{1}^{t-1}, \mathbf{X}_{2}^{t-1}, \cdots, \mathbf{X}_{N}^{t-1}$ at time $t-1$. Since there are some memory cells in the LTM library, to accelerate the convergence speed, randomly select $L$ cells from 
them, that is $A^{t-1}=\left\{\mathbf{X}_{i 1}^{t-1}, \mathbf{X}_{i 2}^{t-1}, \cdots, \mathbf{X}_{i L}^{t-1}\right\}$. To assure the randomness, here we proposed 3 evolution strategies.

\subsubsection{Average evolution}

At time $t$, the state of $\mathbf{X}_{i}$ is transferred to

$$
x_{i j}^{t}=\left\{\begin{array}{l}
\frac{1}{\left|A^{t-1}\right|} \sum_{k \in A^{t-1}} x_{i j}^{t-1}, j \in B \\
x_{i j}^{t-1}, j \in\{1,2, \cdots, n\}-B
\end{array}\right.
$$

where $\mathbf{X}_{i}^{t}=\left(x_{i 1}^{t}, x_{i 2}^{t}, \cdots, x_{i n}^{t}\right), \mathbf{X}_{i}^{t-1}=\left(x_{i 1}^{t-1}, x_{i 2}^{t-1}, \cdots, x_{i n}^{t-1}\right), B=\{1,2, \cdots, n\}$, and $W=\max \{2,(0.1 \% \sim 5.0 \%) n\}$.

\subsubsection{Compound evolution}

To avoid the local minimum and make the evolution more flexible, here we select two alternative solutions from set $A$, the new compound state value is

$$
x_{i j}^{t}=\left\{\begin{array}{l}
\sum_{k=1}^{m_{I}} \alpha_{k} x_{i_{2(k+1)+1} j}^{t-1}-\sum_{k=1}^{m_{E}} \gamma_{k} x_{i_{2 k} j}^{t-1}, j \in B \\
x_{i j}^{t-1}, j \in\{1,2, \cdots, n\}-B
\end{array}\right.
$$

where $m_{I}$ and $m_{E}$ are number of cells, $m_{I} \geq 2, m_{E} \geq 1, m_{I}>m_{E}, L=m_{I}+m_{E}$, $\forall k, s \in\left\{i_{1}, i_{2}, \cdots i_{L}\right\}, k \neq s \neq i, 0<\alpha_{k}, \gamma_{k}<1$.

\subsubsection{Copy evolution}

Obviously there are more than one better alternative solution ( or memory cell $M_{k}$ ), $F\left(\mathbf{X}_{k}^{t-1}\right)<F\left(\mathbf{X}_{i}^{t-1}\right)$, at time $t$, memory cell $M_{i}$ can simply copy the state of $M_{k}$, then the state of $\mathbf{X}_{i}$ is transferred to

$$
x_{i j}^{t}= \begin{cases}x_{k j}^{t-1}, & j \in B \\ x_{i j}^{t-1}, & j \in\{1,2, \cdots, n\}-B\end{cases}
$$

\subsection{The mathematical model for updating and forgetting memory}

At time $t$, assume the memory residual value of memory cell $M_{i}$ is $m_{i}(t)$, then

$$
s_{i}= \begin{cases}I, & m_{i}(t) \leq M_{S} \\ S, & M_{S}<m_{i}(t) \leq M_{L} \\ L, & m_{i}(t)>M_{L}\end{cases}
$$

where $M_{S}$ and $M_{L}$ are critical constant to judge memory state, TM, SM or LM. 


\subsubsection{Model for forgetting memory}

Here we utilize the Ebbinghaus's forgetting curve [13] to model the proceed of memory forgetting, then the memory decaying function of memory cell $M_{i}$ is as follows:

$$
\begin{gathered}
m_{i}(t+\Delta t)=m_{i}(t) e^{-\alpha \Delta t}, \\
\alpha=\left\{\begin{array}{l}
\alpha_{I}, s_{i}=I \\
\alpha_{S}, s_{i}=S \\
\alpha_{L}, s_{i}=L
\end{array}\right.
\end{gathered}
$$

where constant $\Delta t$ is the time increment, memory starts at time $t=0$; coefficient $\alpha$ is used to adjust to memory decaying; For TM, SM and LM, the memory decaying rate $\alpha_{I}, \alpha_{S}, \alpha_{L}$ are different, and as normal, $\alpha_{I}>\alpha_{S}>\alpha_{L}$.

This model is very close to the actual situation of memory decaying, because

$$
\begin{aligned}
& \lim _{\Delta t \rightarrow 0} m_{i}(t) \mathrm{e}^{-\alpha \Delta t}=m_{i}(t) \\
& \lim _{\Delta t \rightarrow \infty} m_{i}(t) \mathrm{e}^{-\alpha \Delta t}=0
\end{aligned}
$$

\subsubsection{Model for updating memory}

At time $t$, memory cell $M_{i}$ accepts a good stimulus and its memory increase $\Delta m_{i}(t)$, the better the stimulus is, the bigger the objective function increment $\Delta F\left(\mathbf{X}_{i}^{t}\right)$ will be, so it is with $\Delta m_{i}(t)$. Here $\Delta m_{i}(t)$ is as follows:

$$
\Delta m_{i}(t)=h\left|\Delta F\left(\mathbf{X}_{i}^{t}\right)\right|
$$

where coefficient $h$ indicates the magnitude of stimulus.

For memory cell $M_{i}$, after the stimulus, its memory will increase to

$$
m_{i}(t+\Delta t)=m_{i}(t)+\Delta m_{i}(t)
$$

Then the real memory becomes to

$$
m_{i}(t+\Delta t)=m_{i}(t) \mathrm{e}^{-\alpha \Delta t}+\lambda m_{i}(t) f_{m}(\Delta t)
$$

where $\lambda$ is the decaying adjustment coefficient, decaying function $f_{m}()$ is used to control memory decaying rate, which suits the Ebbinghaus's forgetting curve.

$$
\lim _{\Delta t \rightarrow \infty} f_{m}(\Delta t)=0
$$

Here assume $f_{m}(x)=\mathrm{e}^{-b x}$ ( $b$ is a constant, $b>\alpha_{L}$ ), then formula (12) above can be written as:

$$
m_{i}(t+\Delta t)=m_{i}(t)\left[\mathrm{e}^{-\alpha \Delta t}+\lambda \mathrm{e}^{-b \Delta t}\right]
$$

Then after memory updating, forgetting and stimulus, the memory updating model of memory cell $M_{i}$ is defined as

$$
m_{i}(t+\Delta t)=m_{i}(t)\left[\mathrm{e}^{-\alpha \Delta t}+\lambda \mathrm{e}^{-b \Delta t}\right]+\Delta m_{i}(t)
$$


Let $\beta=\mathrm{e}^{-\alpha \Delta t}+\lambda \mathrm{e}^{-b \Delta t}$, after $\alpha, b, \Delta t$ are given, $\beta$ is constant and $0<\beta<1$, finally memory function can be rewritten as

$$
m_{i}(t+\Delta t)=m_{i}(t) \beta+\Delta m_{i}(t), \beta=\left\{\begin{array}{l}
\beta_{I}, s_{i}=I \\
\beta_{S}, s_{i}=S \quad, 0<\beta_{L}<\beta_{S}<\beta_{I}<1 \\
\beta_{L}, s_{i}=L
\end{array}\right.
$$

\section{Simulation results}

The computer used to test the performance of AMPO is a Lenovo Notebook computer, its CPU is Intel Core I5, N3450@1.10 GHz, its memory size is 4 GB, its OS is Windows 10.

The setting of parameters used in AMPO are $G=1000, n=15, N=50$, $\beta_{I}=0.55, \beta_{S}=0.35, \beta_{L}=0.15, h=1.2$, $M_{S}=50 h, M_{L}=3 M_{S}, F_{I}=0.7, F_{S}=0.3, F_{L}=0.05, L=3, W=\max \{2,0.01 n\}$, $m_{I}=2, m_{E}=1, \alpha_{1}=1, \alpha_{2}=0.5, P_{A}=1 / 3, P_{B}=2 / 3, P_{C}=1, \gamma_{1}=0.5, \Delta t=0.1$.

We choose two popular optimization algorithms to make comparison with AMPO, they are DE and GA, then choose objective function as $f(\mathbf{X})=t_{f}$, namely to design a trajectory while RLV takes a shortest time to re-entry. The initial states are $h_{0}=120 \mathrm{~km}$, $V_{0}=7200 \mathrm{~m} / \mathrm{s}, \theta_{0}=-1.1^{\circ}$, flight is over when height reaches $28 \mathrm{~km}$. If iteration times reach the max times limits, or objective function value changes less than $0.1 \%$, then we assume that AMPO find the global optima and terminate the iteration.

The algorithms are run independently 100 iterations or 200s, the best objective function value, time of convergence, iteration times, are reported in Table 1 and Table 2.

Table.1 Results of same iterations.

\begin{tabular}{|c|c|c|c|}
\hline Optimization algorithm & Best value & Convergence time & Iterations \\
\hline AMPO & 992.5 & 200.0 & 100 \\
\hline DE & 994.1 & 256.9 & 100 \\
\hline GA & 1074.6 & 360.2 & 100 \\
\hline
\end{tabular}

Table 2. Results of same convergence time.

\begin{tabular}{|c|c|c|c|}
\hline Optimization algorithm & Best value & Convergence time & Iterations \\
\hline AMPO & 992.5 & 200.0 & 100 \\
\hline DE & 1022.7 & 200.0 & 78 \\
\hline GA & 1294.1 & 200.0 & 35 \\
\hline
\end{tabular}

From Table 1 and Table 2 we can see that AMPO successfully converge, it terminate iteration by reaching the maximum of iterations and convergence time limit simultaneously, while DE and GA don't converge. For the same iterations, AMPO's convergence takes less time than DE, and DE takes less time than GA. For the same convergence time, AMPO has less iterations than DE, and DE has less iterations than GA. The results show that AMPO has the ability of high convergence speed, which is very critical for RLV for trajectory design.

Further research shows that if AMPO has a pre-stored LTM library, AMPO will converge faster ( less than 10 seconds ) and makes it possible for online trajectory design.

The results for the trajectory generated by AMPO are shown below. 


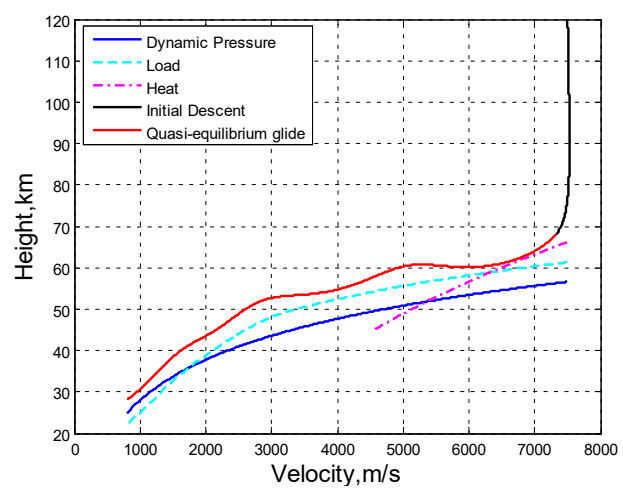

Fig. 2. Trajectory and entry corridor.

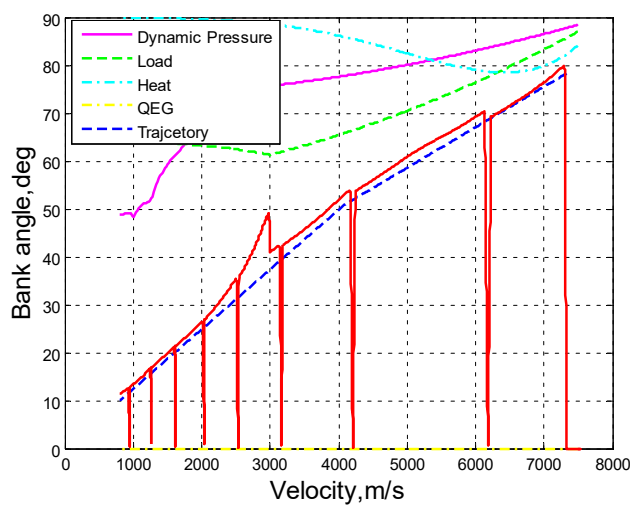

Fig. 4. Velocity history of bank angle.

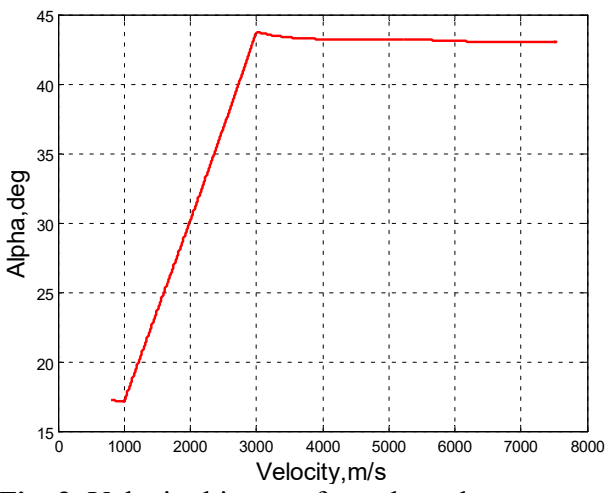

Fig. 3. Velocity history of attack angle.

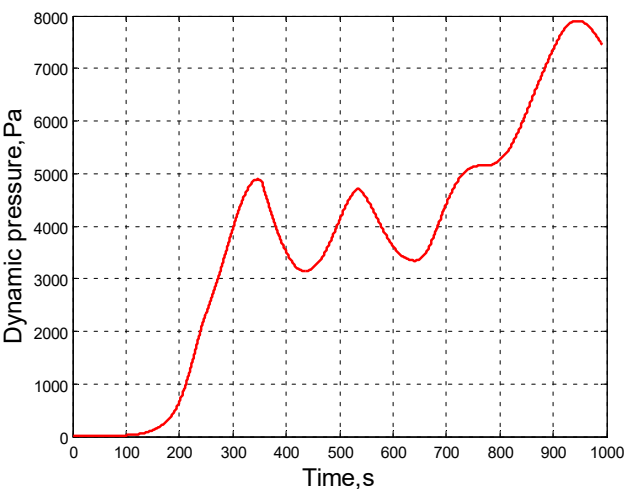

Fig. 5. Time history of dynamic pressur.

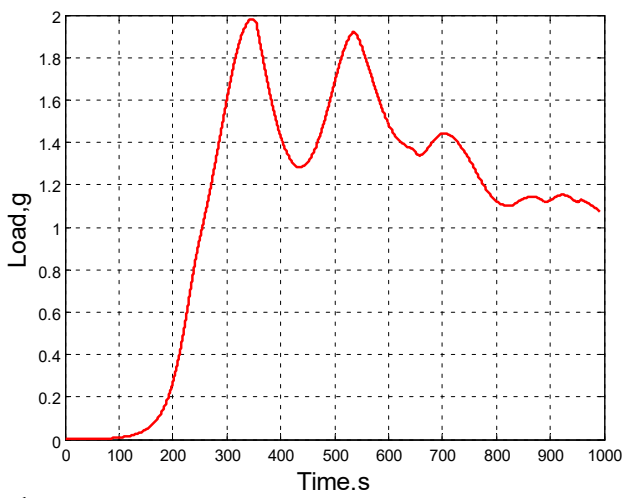

Fig. 6. Time history of load.

Figure 2 gives the entry trajectory in the velocity-altitude space, including the initial decent and phase of Quasi-equilibrium, plus dynamic pressure and load ( normal acceleration ) limits. The trajectory is quite smooth and reliable. Figure 2 shows the angleof-attack ( or alpha ) profile in the alpha-velocity space. Figure 3 shows the bank angle profile in the bank-velocity space, obviously bank angle never exceeds the limits. From Figure 5 and Figure 6 we can find that the maximum dynamic pressure is $7.8 \mathrm{kPa}$ ( less than the limit of $8 \mathrm{kPa}$ ), the maximum load is $1.96 \mathrm{~g}$ ( less than the limit of $2.0 \mathrm{~g}$ ).

\section{Conclusion}


This paper proposed a AMPO algorithm for RLV to design the re-entry trajectory, and make a comparison with DE and GA. Results show that AMPO has a big advantage in speed of convergence, which is quite essential for complicated optimization problems.

The potential drawback of AMPO is that the LTM system requires off-line design of LTM, which works as the initial value for optimization. And AMPO has to improve its speed and flexibility. The concurrent and future work involves the development of evolution strategies that makes AMPO faster, and applicable for online trajectory design.

\section{References}

1. E. M. Yon, L. Chen, G. J. Tang, A survey of numerical algorithms for trajectory optimization of flight vehicles, J. Astro. 29(2008): 398-406.

2. G. Q. Huang, Y. P. Lu, Y. Nan, A survey of numerical algorithms for trajectory optimization of flight vehicles, Sci. China Tech. Sci., 42(2012): 1016-1036.

3. Y. J. Wang, N. H. Xiu, Nonlinear optimization theory, (2012).

4. Y. Nan, G. Q. Huang, Y. P. Lu, Global 4-D trajectory optimization for sapcecraft, Sci China Tech Sci, 53(2010): 2097-2102.

5. Kodera M., Ogawa H., Tomioka S., et al, Multiobjective design and trajectory optimization of space transport systems with RBCC propulsion via evolutionary algorithms and pseudo spectral methods, AIAA SPHST Confer. (2014).

6. Darby C. L., Hager W. W., and Rao, A. V, An hp-adaptive pseudospectral method for solving optimal control problems, Opt. Ctl. Appl. Methods, 32(2011) : 476-502.

7. Stephen J., Dimitri Mavris, Barry Hellman, Rocketback Trajectory Figures of Merit for a Reusable Booster Technology Flight Demonstrator, AIAA SE Confer. (2010).

8. Barry M. H., Anhtuan D. N., Jon W., Technical Challenges for an Integrated Reusable Booster System Flight Demonstrator, AIAA SE Confer. (2010).

9. G. Russo, USV Status 2011: New Steps Ahead, AIAA SPHST Confer. (2011).

10. F.Corraro, G.Morani, F.Nebula, et al, GN\&C Technology Innovations for TAEM USV DTFT2 Mission Results, AIAA SPHST Confer. (2011).

11. C. Chawla, P. Sarmah, R. Padhi, Suboptimal Reentry Guidance of a Reusable Launch Vehicle Using Pitch Plane Maneuver, AST, 14(2010):377-386.

12. G. Q. Huang, T. Li, Q. Q. Lu, Artifitial memory-based optimization, Space Engin. Theo. \& Prac., 34(2014): 2900-2912.

13. Eysenck M. W., Keehn M. T., Cognitive psychology, (2009). 